\title{
LIPIDOMIC PROFILES AS A TOOL TO SEARCH FOR NEW BIOMARKERS
}

\section{ANNA PISARSKA, WOJCIECH WĄSOWICZ, and JOLANTA GROMADZIŃSKA}

\author{
Nofer Institute of Occupational Medicine, Łódź, Poland \\ Department of Biological and Environmental Monitoring
}

\begin{abstract}
Lipidomics belongs to the family of the so-called omics domains, which, based on modern chemical technologies, strive to explain the biological principles of the organism's functioning. Main biological functions of lipids include energy storage, the formation of cell membranes, and participation in the transmission of biological signals, and their dysregulation is responsible for the development of pathological states. Thanks to lipid profiling, potential biomarkers for disease diagnosis and prognosis can be identified. This paper discusses selected examples of the use of lipidomic tests in the diagnosis of the kidney, metabolic and neoplastic diseases based on research papers published over the last few years (since 2016). Only works based on the study of human biological material by mass spectrometry methods were taken into account. The examples of lipidomics application presented in this publication are only a few of the possibilities of this technique. As potential possibilities have already been discovered, the next step for the research community is to work on standardization of the approach to lipidomic research and to develop bioinformatics methods that allow efficient processing and analysis of large amounts of data generated in this technique. Int J Occup Med Environ Health. 2022;35(2):111-26
\end{abstract}

Key words:

cancer, biomarkers, lipids, lipidomics, kidney disease, metabolomic disease

\section{INTRODUCTION}

Lipids form a group of compounds defined by physicochemical properties - they are not soluble in water (they are hydrophobic or amphiphilic) - unlike other classes of biomolecules, which are defined by a common structural feature [1]. Some of the proposed breakdowns of lipids are fatty acids, glycerolipids, glycerophospholipids, sphingolipids, steroids, prenols, sacharolipids, polyketides [2]. The main biological functions of lipids include energy storage, the formation of cell membrane structures, and participation in signal transduction [3].

Lipids belong to the family of the so-called "omics" domains, the purpose of which is to provide information about the current state of the body, as well as to identify new biomarkers of exposure to chemical substances, related to lifestyle (diet), as well as prognostic and diagnostic in selected pathological states [4]. The concept of lipidomic analysis appeared in the world of science at the beginning of the 21st century. The first research papers published in journals date back to 2001 [5]. Since then, a systematic increase in interest in this field has been observed, which translates into the number of articles published (Figure 1). Thanks to the development of technology, new methodological and technical solutions, lipidomics is becoming an attractive branch of metabolomics (Figure 2) [6], which allows learning about cell lipid

Funding: this work was supported by National Sciences Centre Poland (project No. 2017/27/B/NZ5/01922 entitled "Identification of early prognostic metabolomic biomarkers of efficiency of nutritional intervention in hemodialysed patients," grant manager: Prof. Jolanta Gromadzińska) and by Nofer Institute of Occupational Medicine (project No. PNP/IMP/24/2021 entitled "The importance of lipidomic studies in the search for new biomarkers - optimization and validation of the method," grant manager: Anna Pisarska, M.Sc.).

Received: April 15, 2021. Accepted: September 23, 2021.

Corresponding author: Jolanta Gromadzińska, Nofer Institute of Occupational Medicine, Department of Biological and Environmental Monitoring, św. Teresy 8 , 91-348 Łódź, Poland (e-mail: jolanta.gromadzinska@imp.lodz.pl). 


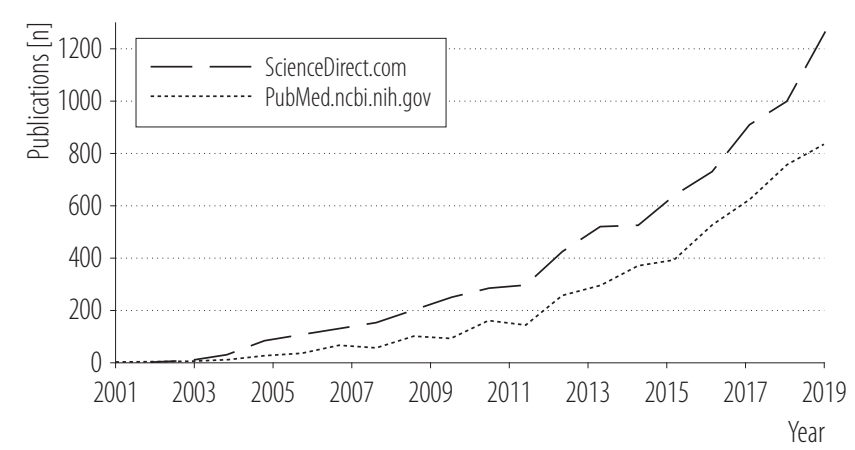

Figure 1. Annual number of publications for "lipidomics analysis" as searched in January 2021

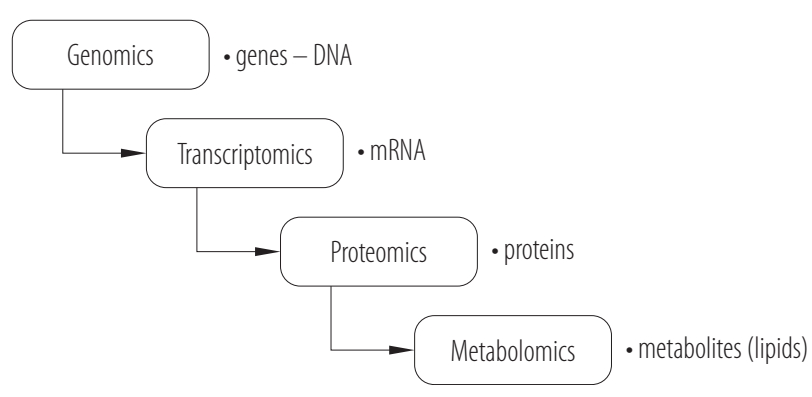

Figure 2. The relationship between genomics, transcriptomics, proteomics and metabolomics

profiles, leading to understanding lifestyle changes, or in physiological and pathological states of the body [7]. The development of this field is therefore particularly important from the point of view of medicine, care for human health, and the need to diagnose adverse changes in the body as early as possible. The definition of lipidomics, created in 2003 and valid until today, is "the full characterization of lipid molecular species and their biological roles with respect to expression of proteins involved in lipid metabolism and function, including gene regulation" [1].

\section{Lipidomic analysis in the diagnosis of diseases}

Research in the field of lipidomics is focused on 5 major branches (Figure 3) [8]. Thanks to lipid profiling, it is possible to track changes in response to changing environmental conditions or the development of pathological

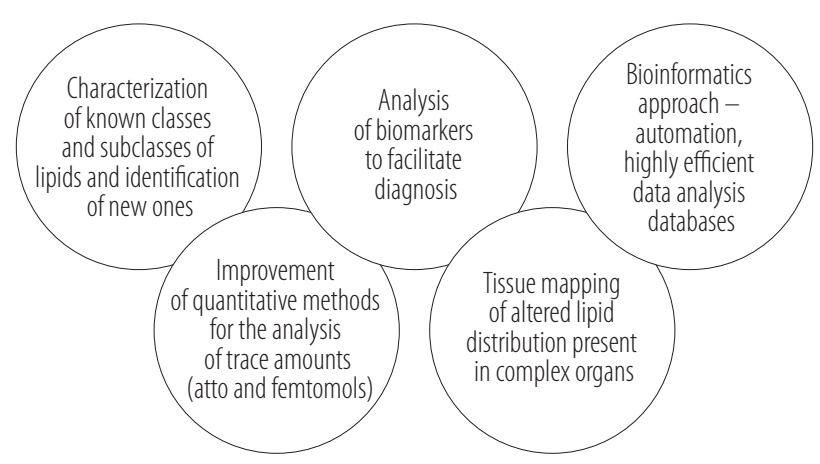

Figure 3. The 5 areas of development of lipidomics

conditions. Linking the lipid composition with changes in the body enables the identification of metabolic pathways. Potential lipid biomarkers can be used in the diagnosis and prognosis of diseases [9]. According to a 2018 report by the World Health Organization (WHO) [10] (presenting data for 2016) among the 10 most common causes of death (causing about $54 \%$ of all deaths) are, among others diabetes, cardiovascular disease, and some types of cancer. The following studies are presented on selected examples, proposing lipid biomarkers, supporting the early diagnosis of selected diseases.

\section{METHODS}

Scientific articles were searched for within the following databases: PubMed and ScienceDirect. For this purpose, the following key words were used: "lipids," "lipidomics," "metabolism," "biomarkers" and accordingly "kidney disease," "cancer" and "diabetes." During the search operators "OR" and "AND" were used. For the analysis, scientific articles published after 2016 were selected. The next step was a detailed analysis of the articles and the selection of those in which analytical base was mass spectrometry. Only works based on the study of human biological material were taken into account. At the end 18 publications were selected the results of which are quoted and analyzed in this study. The background for the entire review was developed on the basis of the remaining 24 publications. 


\section{RESULTS}

\section{Chronic kidney disease}

Epidemiological studies show that the number of cases of kidney disease is constantly increasing in the world, one of which - chronic kidney disease (CKD) - is classified as a lifestyle disease. World data for 2017 shows 697.5 million cases of $\mathrm{CKD}$, resulting in an estimated incidence of $9.1 \%$ in relation to the world population. Also, there were 1.2 million deaths from chronic kidney disease, and a further 1.4 million deaths from cardiovascular disease were attributed to renal impairment. In 1990, CKD was mentioned as the 17th cause of global deaths, in 2017 it was 12 th place. Over the years, the mortality rate also increased - from $35.2 \%$ to $41.5 \%$ [11].

The development of pathological conditions in the kidneys results in a disturbance of the homeostasis, impaired blood filtration and consequently storage of toxins and metabolic products in the body. This starts an avalanche of gradual impairment of the work of subsequent organs. Early detection of CKD may lead to early treatment and consequently may stop the development of the disease in its initial stage (5 are distinguished). In extreme cases, end-stage kidney disease (ESRD) occurs, which requires dialysis or kidney transplantation [12]. Additionally, it causes a great economic burden on the health system. Calculations made by the team of Elshahat et al. [13], based on data collected up to 2015 (published in 2020, adjusted for inflation), indicate that the total costs of treating chronic kidney disease in mild stages (1-3) vary by USD 1600 up to >USD 25000 , and for patients with severe stages (4-5), the cost rises to >USD 56000 . Treating patients with end-stage kidney disease costs >USD 100000 .

Table 1 presents the potential associations of lipids with the prognosis for the occurrence of renal failure. In 2016, a study [14] was conducted to identify prognostic serum lipid metabolites in stages 2 and 3 of chronic kidney disease to predict disease progression to stage 5 . The main criterion to include patients in the study was glomerular filtration rate (eGFR) $<30 \mathrm{ml} / \mathrm{min}$. The study group consisted of a total of 200 patients followed for 6 years, of whom 79 had progression to end-stage renal disease, and in 121 patients without progression, only an eGFR reduction of less than $25 \%$ was observed.

For lipidomic analysis, liquid chromatography was used along with mass spectrometry and a time-of-flight analyzer (LC-TripleTOF-MS). Using the data obtained from the test group, out of 510 initially identified lipids, 49 were qualified (after taking into account statistical methods) for analysis for the prognostic aspect of the development of kidney disease. At the metabolite level, the independent predictors of progression that were most accurate in all 3 classification methods used (partial least squares-discriminant analysis [PLS-DA], false discovery rate [FDR], random forest $[R F]$ ) were diacylglycerols (DAG) (36:0), DAG (32:0), and monoacylglycerol (MAG) (16:0). Diacylglycerols (36:0) and DAG (32:0) were associated with slower disease progression, while MAG (16:0) was associated with faster progression to end-stage renal disease, regardless of the parameters of eGFR and UPCR (urine protein creatinine ratio).

Using the ultra-performance liquid chromatography with a high definition mass spectrometry (UPLC-HDMS), a different profile of metabolites was proposed by an international team of scientists from China and the USA [15]. The metabolic profile of the serum was compared in 180 patients with chronic kidney disease (diagnosed stages $4-5$ ) and 120 healthy people. Patients with acute kidney injury, liver disease, systemic disease as diabetes, lupus erythematous, amyloidosis, patients treated with immunosuppressants in the last 6 months of chemotherapy in the last 2 years, and patients undergoing chronic dialysis or kidney transplantation were excluded.

To find the best combination of significantly altered concentrations of lipid types, several statistical methods were used (including principal component analysis [PCA], or- 
Table 1. Candidate for selective biomarkers of chronic kidney disease in articles published after 2016 collected on Pubmed and ScienceDirect in March 2021

\begin{tabular}{|c|c|c|c|c|c|}
\hline Disease and sample & Lipids and their concentration* & Method & Reference & Test group & Year \\
\hline \multicolumn{6}{|l|}{$\begin{array}{l}\text { Progression to end stage } \\
\text { kidney disease }\end{array}$} \\
\hline serum & $\begin{array}{l}\operatorname{DAG}(36: 0) \downarrow \\
\operatorname{DAG}(32: 0) \downarrow \\
\operatorname{MAG}(16: 0) \uparrow\end{array}$ & LC-TripleTOF-MS & 14 & $\begin{array}{l}79 \text { participants with ESRD } \\
\text { and } 121 \text { without progression }\end{array}$ & 2016 \\
\hline \multicolumn{6}{|l|}{ Chronic kidney disease } \\
\hline serum & $\begin{array}{l}\text { methyl hexadecanoic acid } \uparrow \\
\operatorname{LPC}(24: 1) \uparrow \\
3 \text {-oxooctadecanoic acid } \uparrow \\
\operatorname{PC}(20: 2 / 24: 1) \downarrow\end{array}$ & UPLC-HDMS & 15 & $\begin{array}{l}300 \text { participants } \\
\text { (including } 120 \text { controls) }\end{array}$ & 2017 \\
\hline plasma & $\begin{array}{l}\operatorname{LPC}(18: 2) \downarrow \\
\operatorname{LPC}(20: 3) \downarrow\end{array}$ & $\begin{array}{l}\text { FIA-MS/MS } \\
\text { GC-MS }\end{array}$ & 16 & $\begin{array}{l}77 \text { participants at different stages } \\
\text { of the disease }\end{array}$ & 2019 \\
\hline plasma & saturated C16-C20 free fatty acids $\uparrow$ & LC-MS & 17 & $\begin{array}{l}214 \text { participants at different stages } \\
\text { of the disease }\end{array}$ & 2018 \\
\hline serum & $\begin{array}{l}\operatorname{LPE}(18: 1) \uparrow \\
\operatorname{LPE}(20: 4) \uparrow\end{array}$ & $\begin{array}{l}\text { HPLC-TOF-MS } \\
\text { GC-QqQ/MS }\end{array}$ & 18 & $\begin{array}{l}60 \text { participants } \\
\text { (including } 30 \text { controls) }\end{array}$ & 2019 \\
\hline
\end{tabular}

DAG - diacylglycerol; FIA-MS/MS - flow injection tandem mass spectrometry; GC-MS - gas chromatography combined with mass spectrometry; GC-QqQ/MS - gas chromatography combined with triple quadrupole mass spectrometry; HPLC-TOF-MS - hight-performance liquid chromatography combined with mass spectrometry and a time-of-flight analyzer; LC-MS - liquid chromatography with mass spectrometry; LC-TripleTOF-MS - liquid chromatography with mass spectrometry and time-of-flight analyzer; LPC - lysophosphatidylcholine; LPE - lysophosphatidylethanolamine; MAG - monoacylglycerol; PC - phosphatidylcholine; UHPLC-HDMS - ultra-performance liquid chromatography with a high definition mass spectrometry.

* The levels of potential biomarkers defined as $(\downarrow)$ and $(\uparrow)$ correspondingly represent down- and up-regulation.

thogonal projections to latent structures discriminant analysis [OPLS-DA], analysis of variance [ANOVA], Mann-Whitney test, FDR). Significantly higher concentrations of methylhexadecanoic acid, lysophosphatidylcholine LPC (24:1), and 3-octadecadecanoic acid as well as significantly lower concentrations of phosphatidylcholine PC (20:2/24:1) were strongly correlated with the eGFR and UPCR parameters and were classified by the researchers as the most significant changes in lipid metabolites in patients with CKD. In 2019 an international team of scientists [16] presented the results of their research, the aim of which was to determine the concentration of lipids in the plasma of patients with CKD and patients undergoing hemodialysis (HD).

The study involved 77 people who were divided into 3 groups according to the stage of kidney disease: CKD stage $2-3$, CKD stage $4-5$, hemodialysis patients. The concentrations of phosphatidylcholines (PC), sphingomyelins (SM), and lysophosphatidylcholines (LPC) were determined by flow injection tandem mass spectrometry (FIAMS/MS). Fatty acids (FA) were analyzed as the corresponding fatty acid methyl esters (FAME) using gas chromatography combined with mass spectrometry (GC-MS). Two hundred thirty-nine types of lipids have been identified and 34 of them indicated significant differences between patients in the CKD stage 2-3 and HD groups and 29 between the CKD stage 4-5 and HD groups. Except for 1 case (nonesterified elaidic acid $(\mathrm{C} 18: 1 \omega 9 \mathrm{t})$ ), a reduction in specific lipids was observed in the group of hemodialysis patients. There were no significant differences in LPC concentrations between patients in CKD 2-3 and CKDE 4-5 groups, with 2 exceptions, i.e., LPC (20:3 and 20:4), 
the concentration of which gradually decreased from CKD stage 2-3 to HD.

Patients' health status was assessed after 3 years by analyzing medical records and/or contacting patients or relatives. There was a correlation between increased levels of lysophosphatidylcholine LPC and a better prognosis for slowing disease progression and increasing survival time. Among this group, especially 2 LPC compounds: LPC (18:2) and LPC (20:3), after correcting the values for albumin concentration, were still statistically significantly higher. Also, scientists from the United States published in 2018 [17] the results of their analyzes on the identification of lipid classes are related to changes in acylcarnitine level in all stages of CKD. The team hypothesized that there are dynamic changes in the quantitative composition of plasma lipids in patients at all stages of chronic kidney disease and that these changes are related to impaired lipids catabolic processes ( $\beta$-oxidation). The study included 214 people: 36 in stage 1 or 2 of CKD, 99 in stage 3, 61 in stage 4, and 18 in stage 5 . In total 517 serum lipids detected in positive and negative ion modes and finally concentrations of 330 lipids from 17 different classes were measured using liquid chromatography with mass spectrometry (LC-MS). The key changes observed were greater amounts of saturated C16-C20 free fatty acids (FFAs) in combination with a reduced ratio of long and medium-long acylcarnitines, which are a marker of impaired $\beta$-oxidation, which may contribute to further CKD progression.

In 2019 Polish scientists [18] conducted a study to determine the metabolic pattern based on which it is possible to distinguish healthy patients from those with chronic kidney disease and to determine quantitative and qualitative changes in metabolites between stage 3 and 4 of the disease. The work uses high-performance liquid chromatography combined with mass spectrometry and a timeof-flight analyzer (HPLC-TOF-MS) and gas chromatography combined with triple quadrupole mass spectrometry (GC-QqQ/MS).
Sera collected from 14 patients in CKD stage 3, 16 patients in stage 4 , and 30 healthy people were used for the study. The collected data were subjected to advanced statistical analysis, using, inter alia, PCA analysis, Student's t-test, Mann-Whitney-Wilcoxon test, FDR, p-value and variable importance of projection (VIP), selectivity ratio (SR), ANOVA, or Fisher's least significant differences (LSD). Significantly statistically reduced concentrations of several lysophosphatidylcholines: LPC (16:1), LPC (20:3), and LPC (20:4) were considered potential markers in CKD patients compared to the healthy group. Additionally, a relationship was also observed between the LPC (18:3) concentration and eGFR in the CKD group. Taking into account disease progression, higher concentrations of lysophosphatidylethanolamines LPE (18:1) and LPE (20:4) were considered significant markers in patients with stage 3 and 4 CKD compared to the control group.

\section{Cancer}

In 2018, the Global Cancer Observatory (GCO) database published estimates of global incidence and mortality from 36 types of cancer. The list covered 185 countries. Figure 4 shows the case distribution of the 4 deadliest neoplasms in 2018, distinguishing between women and men [19]. According to the estimates of Pilleron et al. [20] by 2050 , only those aged $\geq 80$ will be diagnosed with $>6.9$ million new cases of cancer (which will constitute $21.5 \%$ of cases in total in all age groups

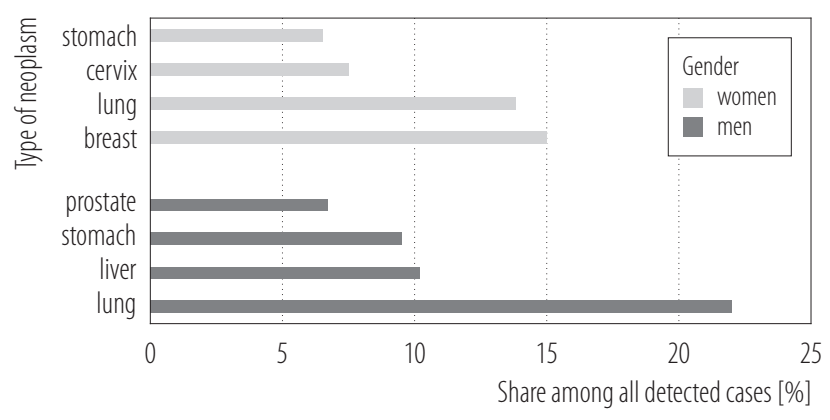

Figure 4. Distribution of the 4 deadliest neoplasm in 2018 
Table 2. Candidate for selective biomarkers of neoplasms in articles published after 2016 collected on Pubmed and ScienceDirect in March 2021

\begin{tabular}{|c|c|c|c|c|c|}
\hline Disease and sample & Lipids and their concentration* & Method & Reference & Test group & Year \\
\hline \multicolumn{6}{|l|}{ Lung cancer } \\
\hline plasma & $\begin{array}{l}\operatorname{LPE}(18: 1) \uparrow \\
\mathrm{ePE}(40: 4) \uparrow \\
\mathrm{CE}(18: 2) \downarrow \\
\mathrm{SM}(22: 0) \downarrow\end{array}$ & ESI-MS & 21 & $\begin{array}{l}346 \text { samples (including } \\
113 \text { adenocarcinomas, } 86 \text { squamous } \\
\text { cell carcinoma, } 147 \text { controls) }\end{array}$ & 2017 \\
\hline serum & $\operatorname{LPC}(18: 2) \downarrow$ & MALDI-TOF-MS & 22 & 400 samples (including 300 controls) & 2017 \\
\hline serum & $\begin{array}{l}\mathrm{FA}(20: 4) \downarrow \\
\mathrm{FA}(22: 0) \downarrow \\
\operatorname{LPE}(20: 4) \uparrow\end{array}$ & UHPLC-Q-TOF/MS & 23 & 127 samples (including 63 controls) & 2020 \\
\hline plasma & $\begin{array}{l}\text { TG }(50: 1) \uparrow \\
\text { TG }(54: 4) \uparrow \\
\operatorname{PE}(38: 3) \downarrow \\
\operatorname{PE}(18: 1 p / 20: 4) \downarrow \\
\text { SM (d18:1/20:0) } \downarrow\end{array}$ & UHPLC-ESI-MS/MS & 24 & 37 samples (including 20 controls) & 2019 \\
\hline \multicolumn{6}{|l|}{ Liver cancer } \\
\hline plasma & $\begin{array}{l}\mathrm{PI}(16: 0 / 18: 2) \uparrow \\
\mathrm{PI}(16: 0 / 20: 4) \uparrow \\
\mathrm{PI}(18: 0 / 20: 3) \uparrow \\
\mathrm{DAG}(16: 1 / 18: 0) \uparrow\end{array}$ & UHPLC-ESI-MS/MS & 24 & 41 samples (including 20 controls) & 2019 \\
\hline \multicolumn{6}{|l|}{ Gastric cancer } \\
\hline plasma & $\begin{array}{l}\mathrm{PC}(36: 3) \downarrow \\
\mathrm{PC}(36: 4) \downarrow \\
\mathrm{LPA}(18: 2) \downarrow\end{array}$ & UHPLC-ESI-MS/MS & 24 & 40 samples (including 20 controls) & 2019 \\
\hline \multicolumn{6}{|l|}{ Breast cancer } \\
\hline tissue samples & $\begin{array}{l}\mathrm{TG}(\mathrm{m} / \mathrm{z} 879.8) \downarrow \\
\mathrm{TG}(\mathrm{m} / \mathrm{z} 881.8) \downarrow \\
\mathrm{TG}(\mathrm{m} / \mathrm{z} 907.8) \downarrow \\
\mathrm{PC}(\mathrm{m} / \mathrm{z} 782.6) \uparrow \\
\mathrm{PC}(\mathrm{m} / \mathrm{z} 725.6) \uparrow \\
\mathrm{PC}(\mathrm{m} / \mathrm{z} 756.6) \text { ions } \uparrow\end{array}$ & $\begin{array}{l}\text { FNA combined with } \\
\text { MALDI-TOF/MS }\end{array}$ & 25 & $\begin{array}{l}8 \text { samples (in pair tumor } \\
\text { and normal tissues) }\end{array}$ & 2017 \\
\hline plasma & $\begin{array}{l}\text { panel of } 15 \text { plasma lipid }-4 \mathrm{LPC} \downarrow \\
6 \mathrm{PC} \uparrow \\
2 \mathrm{ePC} \uparrow \\
3 \mathrm{CE} \text { species } \downarrow\end{array}$ & LC-ESI-MS/MS & 26 & $\begin{array}{l}194 \text { samples ( } 84 \text { with early-stage breast } \\
\text { cancer and } 110 \text { with benign disease) }\end{array}$ & 2016 \\
\hline \multicolumn{6}{|l|}{ Cervix cancer } \\
\hline plasma & $\begin{array}{l}\mathrm{PC}(18: 1 / 15: 0) \downarrow \\
\mathrm{PC}(18: 2 / 20: 5) \downarrow \\
\operatorname{LPC}(18: 0) \uparrow \\
\operatorname{LPC}(10: 0) \uparrow\end{array}$ & UPLC-MS & 27 & $\begin{array}{l}182 \text { samples ( } 89 \text { with cervical cancer } \\
\text { and } 93 \text { with uterine fibroids) }\end{array}$ & 2016 \\
\hline $\begin{array}{l}\text { Prostate cancer } \\
\text { urine (urinary } \\
\text { exosomes) }\end{array}$ & $\begin{array}{l}\text { LacCer (d18:1/16:0) } \\
\text { PS (18:1/18:1) } \downarrow \\
\text { PS (18:0/18:2) } \uparrow\end{array}$ & LC-MS/MS & 28 & 28 samples (including 13 controls) & 2017 \\
\hline
\end{tabular}


Table 2. Candidate for selective biomarkers of neoplasms in articles published after 2016 collected on Pubmed and ScienceDirect in March 2021 - cont.

\begin{tabular}{|c|c|c|c|c|c|}
\hline Disease and sample & Lipids and their concentration* & Method & Reference & Test group & Year \\
\hline \multicolumn{6}{|l|}{ Prostate cancer - cont. } \\
\hline tissue & $\begin{array}{l}\text { linoleic acid } \uparrow \\
\text { arachidonic acid } \uparrow \\
\text { oleic acid } \uparrow\end{array}$ & GC-MS & 29 & $\begin{array}{l}102 \text { samples } \\
\text { (including } 50 \text { benign prostatic hyperplasia } \\
\text { as control) }\end{array}$ & 2021 \\
\hline
\end{tabular}

CE - cholesteryl esters; DAG - diacylglycerol; ePC - ether-linked phosphatidylcholine; ePE - phosphatidylethanolamine (PE) with one ether-linked (alkyl or alkenyl) chain; ESI-MS - electrospray ionisation mass spectrometry; FA - fatty acid; FNA - fine needle aspiration; GC-MS - gas chromatography; LC-ESI-MS/MS - liquid chromatography with tandem triple quadrupole mass spectrometry with electrospray ionization; LC-MS/MS - liquid chromatography with tandem mass spectrometry; LecCer - lactosylceramide; LPA - lysophosphatidic acid; LPC - lysophosphatidylcholine; LPE - lysophosphatidylethanolamine; MALDI-TOF-MS - matrix-assisted laser desorption/ionization mass spectrometry with time-of-flight analyzer; PC - phosphatidylcholine; PE - phosphatidylethanolamine; PI - phosphatidylinositol; PS - phosphatidylserine; SM - sphingomyelin; TG - triacylglycerol; UHPLC-ESI-MS/MS - ultra-efficient liquid chromatography with electrospray ionization and tandem mass spectrometry; UHPLC-Q-TOF/MS - ultra-high performance liquid chromatography in combination with quadrupole mass spectrometry with time-of-flight analyzer; UPLC-MS - ultra-performance mass spectrometry liquid chromatography.

* The levels of potential biomarkers defined as $(\downarrow)$ and $(\uparrow)$ correspondingly represent down- and up-regulation.

in the world), which would be an increase of $200 \%$ compared to 2018 data. Undoubtedly, early diagnosis could inhibit predicting such a dramatic increase in the incidence. Table 2 presents selected examples of the relationship between a given disease entity and lipids, the changes in the concentration of which in the samples was observed during the research, thanks to which they can probably be used in cancer prognosis.

In 2017 the team of Yu et al. [21] used lipidomics to identify markers of lung cancer. In the study, plasma samples from 199 patients with early-stage non-small cell lung cancer (NSCLC), including 113 adenocarcinomas and 86 cases of squamous cell carcinoma, were profiled by mass spectrometry. The control consisted of 147 plasma samples collected from healthy people. In the first stage, after the identification of 361 lipids in the samples, those for which there was evidence of low clinical usefulness were excluded, e.g., due to very low concentrations $(<10 \mathrm{nmol} / \mu \mathrm{l}$ in plasma) or their slight differences between patients and healthy people $(\leq 10 \%)$. After the evaluation of the diagnostic value for the obtained data (i.e., sensitivity $\geq 80 \%$, specificity $\geq 50 \%$, receiver operating characteristic $[\mathrm{ROC}] \geq 80 \%$ ), no lipid meeting the required criteria was identified. The use of a combined biomarker strategy allowed to define a panel of lipids that are candidate biomarkers for the early detection of NSCLC disease - LPE (18:1), phosphatidylethanolamine with 1 ether-linked (alkyl or alkenyl) chain PE (40:4), cholesteryl ester CE (18:2) and SM (22:0). The limitation of this study is the lack of analyzes of samples of benign lung tumors, which would be additional confirmation for the proposed panel of lipid biomarkers.

In the same year, a team of researchers from Poland [22] used matrix-assisted laser desorption/ionization mass spectrometry with a time-of-flight analyzer (MALDITOF-MS) to analyze serum samples from 100 patients with early-stage lung cancer (including 31 cases detected by screening) and from an age and gender-matched group of 300 healthy participants in a lung cancer screening program to determine tumor-specific lipid profiles. The analysis revealed 209 lipids differentiating tumor and control samples. After using statistical tools, (including Bonferroni correction) for multiple comparison, 59 components retained statistically significant differences. Lysophosphatidylcholine LPC (18:2), LPC (18:1), and LPC (18:0) concentrations were significantly lower in the serum of cancer patients. A complementary approach was introduced using LC-MS in samples from 100 cancer 
patients and 100 controls, confirming the previously obtained results.

Based on the collected data, it was found that satisfactory results to which it is possible to distinguish sick people from healthy people are obtained based on the concentration of LPC alone (18:2). The MALDITOF-MS used in the above-mentioned study made it possible to detect tumor-specific serum lipid profiles, but a direct identification of all their types was not possible due to the relatively low mass resolution in the technique used.

Lung cancer biomarkers were also investigated by the team of Noreldeen et al. [23] using non-targeted lipidomic profiling based on ultra-high performance liquid chromatography in combination with quadrupole mass spectrometry with a time-of-flight analyzer (UHPLC-QTOF/MS). A study was conducted to define more than 1 lipid metabolite in serum samples of non-small cell lung cancer patients. A total of 127 women was participating in the study, and 63 of them were in control. The total number of lipid ions detected was 276 and 260 in negative and positive mode, respectively. After taking into account statistical methods (including PCA, PLS-DA, MannWhitney U test), a total of 79 lipid ions showed statistically significant differences between women diagnosed with lung cancer and controls. The data indicated that in serum of non-smoking women with non-small cell-lung cancer 12 individuals is promising biomarkers, especially in the metabolism of fatty acids and lysophosphatidylethanolamines - FA (20:4), FA (22:0), and LPE (20:4). The concentration of FA (20:4) was always lower, while FA (20:0) and LPE (20:4) showed always higher levels in patients with early-stage lung cancer than in the control group. The authors suggest that the combined analysis of the concentrations of the 3 metabolites mentioned may serve as a sensitive and specific biomarker to distinguish NSCLC patients from healthy individuals (also to patients with early-stage cancer).
South Korean researchers [24] analyzed plasma samples from 58 cancer patients of various locations (including liver, stomach, lung cancer) to determine the differences in lipid metabolism between these types of cancer compared to 20 healthy subjects. Only male samples were used in the study to exclude possible hormonal effects on the regulation of blood lipid metabolite levels. The analysis was performed using ultra-efficient liquid chromatography with electrospray ionization and tandem mass spectrometry (UHPLC-ESI-MS/MS). The use of nontargeted lipidomics allowed to identify of 335 lipids, among which 50 were selected, present in a concentration 2 times higher $(\mathrm{p}<0.01)$ in at least one of the tumors as compared to the control. The number of lipids considered significant in the 3 types of cancer was 8 , in 2 types it was already 15.

Further analysis allowed for the identification and selection of lipids specific for each tumor:

- liver: phosphatidylinositoles PI (16:0/18:2), PI (16:0/ 20:4), PI (18:0/20:3) and diacylglycerol DAG (16:1/18:0); - stomach: phosphatidylcholines PC (36:3) and PC (36:4) and lysophosphatidic acid LPA (18:2);

- lungs: triacylglyceroles TG (50:1) and TG (54:4), phosphatidylethanoloamines PE (38:3) and PE (18:1p/20:4) and sphingomyelin SM (d18:1/20:0).

Researchers in Taiwan [25] proposed a complementary cytology test technique to improve the accuracy of breast cancer diagnoses by detecting lipid biomarkers. The study used tissues collected during a fine needle aspiration (FNA), from which the lipid fraction was then extracted and characterized by mass spectrometry using the MALDI-TOF technique. Tissue samples were collected during surgical operations in pairs - tumor and normal tissue. To avoid interference from heme ( $\mathrm{m} / \mathrm{z} 616)$ that is released from hemoglobin in tissue samples during the biopsy, the molecular profile was narrowed down to the range of 700-1000 Da. Very high intensity of TG ion signals ( $\mathrm{m} / \mathrm{z} 879.8, \mathrm{~m} / \mathrm{z}$ 881.8, and $\mathrm{m} / \mathrm{z}$ 907.8) 
- was detected in the area of tissue not affected by neoplastic lesions, and the reverse trend of TG ion signals was found in the area of neoplastic tissue. In contrast, very strong phosphatidylcholines PC ion signals $(\mathrm{m} / \mathrm{z} 782.6$, $\mathrm{m} / \mathrm{z} 725.6$, and $\mathrm{m} / \mathrm{z} 756.6$ ) were detected in the area of neoplastic tissue and low intensities in unchanged control tissues. Lipid biomarkers, proposed in this study were identified in intraoperatively harvested breast tissues. The idea behind this study was to develop a strategy to support biopsy by identifying molecular biomarkers during initial diagnosis.

A joint team of scientists from China and the USA had a different approach to the use of lipidomic tests related to breast cancer diagnosis [26]. A study was carried out to identify a lipid panel that could distinguish early-stage breast cancer from benign lesions and serve as potential biomarkers in the diagnosis of breast cancer. A total of 194 plasma samples from 84 women with early-stage breast cancer (stage $0-\mathrm{II}$ ) and 110 patients with benign tumors were analyzed using tandem triple quadrupole mass spectrometry with electrospray ionization (LCESI-MS/MS). From the initial number of 367 lipids, after the introduction of restrictions on, inter alia, the minimum concentration in the samples was reduced to 191. Then, after taking into account the statistical significance and the Student's t-test, a total of 15 lipids (lysophosphatidylcholines LPC (18:3), LPC (20:2), LPC (20:1), LPC (20:0), cholesteryl esters C (19:1) CE, C (19:0) CE, C (20:0) CE, phosphatidylcholines PC (32:1), PC (34:4), PC (38:3), PC (40:5), PC (40:3), PC (44:11), ether-linked phosphatidylcholine PC (32:2), ePC (38:3)) have been identified as potential biomarkers to help diagnose breast cancer.

When using binary logistic regression to build a predictive model, it was found that single groups of lipids did not give good diagnostic results and it was necessary to consider all 15 lipids simultaneously. The limitations of this study consisted in the large diversity among the benign breast tumors studied (including hyperplasia, fibroadenoma, cysts, and other unspecified changes), low race differentiation ( $>90 \%$ were Caucasian), and the inability to correlate changes in potential lipid biomarkers with the size of tumors (due to the lack of information about their size).

Given the steadily increasing number of cervical cancer cases in developing countries, an international team led by Yin et al. [27] conducted an ultra-performance mass spectrometry liquid chromatography (UPLC-MS) non-targeted analysis of plasma samples from 89 patients diagnosed with cervical cancer (SCC) and 93 controls from patients with uterine fibroids (UF). Using the multivariate statistical analysis of PLS-DA, 10 lipid metabolites with significantly different levels of expression were selected between patients with SCC and UF. Using an area under the curve (AUC) ratio $\geq 0.75$, 4 potential tumor biomarkers were selected phosphatidylcholines PC (18:2/20:5) and PC (18:1/15:0), lysophosphatidylcholines LPC (10:0) and LPC (18:0).

To further validate the results obtained with the chromatographic method, the concentrations of phosphatidylcholine and lysophosphatidylcholine in the plasma of patients with SCC and UF were tested with ELISA tests. Analogous differences were obtained - the concentration of PC was significantly lower in the serum of patients with cervical cancer compared to the controls, the relationship was reversed in the case of LPC.

The obtained results suggest that changes in the concentration of selected lipids can be used as potential biomarkers for the diagnosis of cervical cancer. The best values of sensitivity, specificity, and AUC were achieved considering all 4 metabolites simultaneously. Similar incidence statistics are represented by cervical cancer in women and prostate cancer in men.

A Scandinavian team of scientists has been looking for potential biomarkers in prostate cancer [28]. In a study conducted on 28 samples (including 13 from healthy subjects and 15 diagnosed with prostate cancer) by liquid chromatography with tandem mass spectrometry 
(LC-MS/MS), 107 lipids were identified and quantified in urine exosome samples. To verify the repeatability of the method, 2 samples were taken (in 2 consecutive days), tested twice from healthy people. The samples taken showed slight quantitative differences (day-to-day variation) mainly within sphingomyelins and glycosphingolipids. Due to the limitations resulting from the size of the samples, the analysis of 36 lipids based on their concentration in urine exosomes was focused on, thanks to which 9 metabolites differing significantly between the compared groups were selected.

Statistically significant differences were shown for lactosylceramide (LacCer) (d18:1/16:0), phosphatidylserines PS (18:1/18:1) and PS (16:0/18:1). The achieved sensitivity oscillated between $67-53-33 \%$, respectively, which means unsatisfactory diagnostic efficiency of individual lipids. Nevertheless, promising results were obtained by analyzing the combination of the lipids mentioned based on mutual ratios - LacCer (d18:1/16:0):PS (18:1/18:1) and PS (18:0/18:2):PS (18:1/18:1). By creating 2 pairs of metabolites, a sensitivity of $93 \%$ and a specificity of $100 \%$ were obtained.

In a study published in 2021, Polish scientists [29] confirmed that in the case of prostate cancer, changes in fatty acid concentrations were observed. Non-targeted lipidomic analysis by gas chromatography coupled with mass spectrometry (GC-MS) were used. Formalin-fixed, paraffin-embedded (FFPE) tissue samples from 52 patients were used. Benign prostatic hyperplasia samples $(\mathrm{N}=50)$ were used as control. Finally, a set of 20 metabolites was obtained and used for statistical analysis. Of these, 11 were selected using one-dimensional statistical analysis. Ultimately, the application of multivariate statistics allowed to distinguish 3 metabolites of linoleic acid, arachidonic acid and oleic acid as the most important for studied samples. An increased level of those fatty acids was observed in the prostate cancer samples. One of the important aspects of the proposed work is the use of samples of histo- logical preparations in lipidomics. In this type of material, the molecular changes associated with cancer development can be followed similarly to fresh tissue, while reducing the challenges of sample availability and storage.

\section{Metabolic diseases}

The term "metabolic disease" defines any disorders that cause disturbances in several biochemical processes taking place in the body and the accompanying energy transformations [30]. There is a significant level of error tolerance in metabolic activities - the disease will only occur when the critical enzyme is turned off and there is no competitor to it that could participate in modifying the same molecule [31]. Diabetes is one of the most common metabolic diseases today.

Diabetes mellitus is characterized by chronic hyperglycemia with disturbances in carbohydrate, fat, and protein metabolism resulting from a defect in insulin secretion and/or function. Chronic hyperglycemia is associated with damage, dysfunction, and even failure of many organs, in particular, the eyes, kidneys, nerves, heart, and blood vessels [32,33]. According to a WHO report, in 1980-2014 the number of people suffering from diabetes increased from 108 to 422 million [34]. The World Diabetes Federation predicts that the incidence will reach 700 million by 2045 [35]. The WHO classification of diabetes mellitus distinguishes 3 main types of diabetes: type 1 , type 2 and gestational diabetes, as well as other specific types of diabetes.

Type 2 diabetes accounts for $90-95 \%$ of all diagnosed diabetes cases. The risk factor of type 2 diabetes is obesity, smoking, unhealthy diet, lack of exercise, a genetic burden. In the early stages of the disease, no insulin is needed, and there are more options for treatment than for type 1 , such as the use of drugs, proper diet, and exercise [36]. In 2019, a group of scientists led by Dall et al. [37] presented a breakdown of the costs incurred as a result of health care (USD 302 billion) and reduced professional 
Table 3. Candidate for selective biomarkers of diabetes mellitus in articles published after 2016 collected on Pubmed and ScienceDirect in March 2021

\begin{tabular}{|c|c|c|c|c|c|}
\hline Disease and sample & Lipids and their concentration* & Method & Reference & Test group & Year \\
\hline \multicolumn{6}{|l|}{ Prediabetes and obesity } \\
\hline serum & $\begin{array}{l}\text { SM (18:0) in prediabetes } \uparrow \\
\operatorname{LPC}(17: 0) \downarrow \\
\operatorname{LPC}(18: 1) \downarrow \\
\operatorname{LPC}(18: 2) \text { correlates with } B M I \downarrow\end{array}$ & LC- and FIA and ESI-MS/MS & 38 & 64 participants & 2016 \\
\hline \multicolumn{6}{|l|}{ Type 2 diabetes risk } \\
\hline serum & $\begin{array}{l}\text { ratio } \operatorname{LPC}(18: 2): \operatorname{LPE}(18: 2) \uparrow \\
\text { phospholipid precursors } \\
\text { (glycerol-2- and 3-phosphates) } \downarrow \\
\text { LPE (18:2) } \downarrow\end{array}$ & GC-TOF-MS and LC-TOF-MS & 39 & $\begin{array}{l}75 \text { participants } \\
\text { (13-year follow-up) }\end{array}$ & 2019 \\
\hline plasma & $\begin{array}{l}5 \text { biomarkers including } \\
\operatorname{LPC}(18: 1) \downarrow\end{array}$ & UPLC-QTOF-MS & 41 & $\begin{array}{l}108 \text { participants } \\
\text { (10-years follow-up) }\end{array}$ & 2017 \\
\hline \multicolumn{6}{|l|}{$\begin{array}{l}\text { Hyperlipidemia } \\
\text { associated with } \\
\text { type } 2 \text { diabetes }\end{array}$} \\
\hline \multirow[t]{2}{*}{ plasma } & $\begin{array}{l}13 \text { glycerophospholipids }-11 \uparrow / 2 \downarrow \\
11 \text { sphingolipids } \uparrow \\
9 \text { glycerolipids }-6 \uparrow / 3 \downarrow \\
3 \text { prenol lipids } \uparrow \\
1 \text { saccharolipid } \uparrow\end{array}$ & UPLC-QTOF-MS & 42 & $\begin{array}{l}79 \text { participants ( } 36 \text { - patients } \\
\text { with hyperlipidemia associated } \\
\text { with type } 2 \text { diabetes; } 43 \text { controls) }\end{array}$ & 2021 \\
\hline & $\begin{array}{l}10 \text { glycerophospholipids } 8 \uparrow / 2 \downarrow \\
7 \text { sphingolipids } \uparrow \\
3 \text { prenol lipids } \uparrow \\
2 \text { glycerolipids } \downarrow\end{array}$ & & & $\begin{array}{l}77 \text { participants ( } 36 \text { - patients } \\
\text { with hyperlipidemia associated } \\
\text { with type } 2 \text { diabetes; } 41 \text { patients } \\
\text { with type } 2 \text { diabetes) }\end{array}$ & \\
\hline
\end{tabular}

ESI-MS/MS - electrospray ionization tandem mass spectrometry; FIA-MS/MS - tandem mass spectrometry with flow injection analysis; GC-TOF-MS - gas chromatography combined with a time-of-flight analyzer and mass spectrometer; LC-MS/MS - liquid chromatography coupled with tandem mass spectrometry; LC-TOF-MS - liquid chromatography combined with a time-of-flight analyzer and mass spectrometer; LPC - lysophosphatidylcholine; LPE - lysophosphatidylethanolamine; SM - sphingomyelin; UPLC-QTOF-MS - quadrupole time-of-flight mass spectrometry analyzer.

* The levels of potential biomarkers defined as $(\downarrow)$ and $(\uparrow)$ correspondingly represent down- and up-regulation.

activity (USD 102 billion) related to diabetes, based on the example of the United States. The total annual burden of USD 404 billion translates into an additional financial burden of an average of USD 1240 per American. These calculations clearly show the enormous scale of the problem, both from the medical and economic point of view, to be faced. Table 3 presents the potential associations of lipids with prognosis for diabetes.
In 2016, a group of scientists from Spain [38] conducted a study involving 64 people classified into 4 groups in terms of body mass index (BMI) and the risk of developing type 2 diabetes (determined based on fasting plasma glucose and insulin resistance). Forty-five women and 19 men participated in the study, but no significant influence of gender on the results was noticed. AbsolutelDQ ${ }^{\circledR}$ 180 Kit, liquid chromatography coupled with LC-MS/MS, 
and tandem mass spectrometry with flow injection analysis (FIA-MS/MS) were used in the work. The remaining metabolites (beyond the detectability of the AbsolutelDQ ${ }^{\circledR} 180 \mathrm{Kit}$ ) were quantified by electrospray ionization tandem mass spectrometry (ESI-MS/MS).

After introducing statistical limitations (metabolites below the quantification limit set at 25\% and with high analytical variability in relation to replicates, $>25 \%$, were rejected), 246 metabolites were taken into account for further analysis. The altered concentrations of the 3 lysophosphaditylcholine, LPC (17:0), LPC (18:1), and LPC (18:2), were strongly negatively correlated with BMI, and thus these compounds are the specific metabolites associated with morbid obesity but not with prediabetes. Higher level of sphingomyelin SM (18:0) and lower of SM (18:1) in prediabetic patients were confirmed to be candidate for the selective markers of prediabetes. However, increased concentration of several forms of ceramides (C17, d18:1/18:0) and dihydroceramides (C20:0, C22:0, C24:1) were selected as putative metabolic markers of obesity and pre-diabetes. Selected metabolites analyzed together as 1 complex potential biomarker achieved an error in the correct classification of people $>53 \%$. By considering obesity and pre-diabetes separately, predictability improved significantly, especially in morbid obesity.

Other potential biomarkers were proposed in 2019 [39] based on a 13-year prospective cohort study in black women in southern Africa with normal glucose tolerance. Ultimately, 75 women participated in the study, 20 of whom developed type 2 diabetes, 27 had impaired glucose tolerance, and 28 were still normal glucose tolerant. Metabolic analyzes were performed using gas and liquid chromatography combined with a time-offlight analyzer and mass spectrometer (GC-TOF-MS and LC-TOF-MS in positive and negative ion mode). The combination of these 2 techniques allowed the detection of $>1000$ putative metabolites in serum samples, of which 252 were identified and classified. Before diagnosis, the group who later developed type 2 diabetes had a lower concentration of LPE (18:2), a higher ratio of LPC concentrations (18:2) to LPE (18:2), and lower concentrations of phospholipid precursors (glycerol-2and 3-phosphates), compared to the group with normal glucose tolerance.

Similar observations were made in the 2012 study [40], where a reduced LPC (18:2) was the marker of both prediabetes and type 2 diabetes in the 2 KORA and EPIC cohorts, which included Caucasians.

The creation of a panel of biomarkers for the development of type 2 diabetes was also aimed at a study published in 2017 [41] in which 108 subjects with established pre-diabetes were followed for 10 years for either developed diabetes or regression to normal glucose regulation. Non-targeted analysis of fasting plasma samples using UPLC-QTOF-MS was used. Among the subjects, 68 subjects remained pre-diabetic, 20 subjects had a regression to the state of normal glucose tolerance, and the same number of subjects developed diabetes. At baseline, there were no significant differences (including age, gender, body mass index, general health, and blood glucose levels) between study participants. In the final stage of the study, differences in 3 parameters were observed: fasting glucose concentration after $2 \mathrm{~h}$ in the test of glucose load and the concentration of glycosylated hemoglobin $\left(\mathrm{HbA}_{1 \mathrm{c}}\right)$. Based on the obtained data, 23 and 22 compounds were selected, respectively, involved in biochemical metabolic pathways of high statistical significance related to regression to normal glucose tolerance or the development of diabetes mellitus. The key to this study was the selection and combination of specific biomarkers to establish a non-invasive and accurate method of predicting prognosis before developing diabetes.

After taking into account the statistical analysis, 5 biomarkers were selected, including amino acid and lipid 
metabolites, the combination of which can be used to predict the development of diabetes - isovaleric aldehyde, linoleic acid, LPC (18:1), 2-pyrroloylglycine, dityrosine, and 20-hydroxy-leukotriene E4, LPC (20:4), 5-methoxytryptamine, endomorphine-1, LPC (20:3) as markers of return to normal glucose tolerance.

Scientists from the University of Zhengzhou (China) [42] used non-targeted lipidomic to study the differences in lipid profiles between 3 groups:

- patients with hyperlipidemia associated with type 2 diabetes (T2D HL) $(\mathrm{N}=36)$,

- patients with type 2 diabetes - T2D $(\mathrm{N}=41)$,

- healthy people $(\mathrm{N}=43)$.

For this purpose, ultra-performance liquid chromatography was used in conjunction with a quadrupole timeof-flight mass spectrometry analyzer (UPLC-QTOF/ MS) for plasma analysis. While processing the results, the PCA analysis and the OPLS-DA analysis were used. Compared with the healthy control group, 37 lipids were significantly altered in the T2D HL group, and when compared with the T2D group, 22 lipids were significantly altered in the T2D HL group. Of all the lipid categories detected, which included sphingolipids, glycerolipids, glycerophospholipids, prenol lipids and the largest proportion in both groups accounted for sphingolipids and glycerophospholipids. In the T2D HL group compared to healthy controls 32 lipids showed higher concentration and only 3 glycerolipids and 2 glycerophospholipids showed lower concentration. Similarly, in T2D HL group compared to T2D group, 18 lipids showed higher concentration, 2 glycerolipids and 2 glycerophospholipids had lower concentration. The identified lipids may provide a deeper understanding of lipid metabolic changes and the relevant metabolic pathway of type 2 diabetes-related hyperlipidemia, which may be useful for improving the risk prediction of this condition.

The above summaries (Tables 1-3) show how the monitoring of the concentrations of individual lipids or their mutual relations can contribute to the identification of potential disease biomarkers. Despite the challenges that undoubtedly include an individual course of the disease, a huge variety of lipid compounds present in body fluids, or laborious preparation of samples preceding the analysis process, advances in the field of lipidomics open the door to more accurate and precise diagnosis of diseases.

\section{CONCLUSIONS}

Despite being a young technique, lipidomics is a powerful tool in biomedical sciences. Its main advantage is showing the mechanisms responsible for pathological conditions in the body, which results from the very nature of this field - comprehensive analysis (identification and quantification) of hundreds of types of lipids. However, like any emerging field, lipidomics has barriers that scientists around the world are just learning to overcome. They include, among others the amount and complexity of known lipids, the difficulty of applying a uniform preparation to such a numerous group of compounds that differ in terms of physicochemical properties, or the need to implement computational bioinformatics. The answers to these needs are numerous initiatives, such as the LipidMaps, LipidBlast, LipidBank platforms containing data on lipids (e.g., their structures or properties) and organizations associating scientists such as the International Lipidomics Society (ILS), whose aim is to promote lipidomics and global cooperation for the development of this technique.

The examples of lipidomics application presented in this publication are only a few of the possibilities of this technique. As potential possibilities have already been discovered, the next step for the research community is to work on standardization of the approach to lipidomic research and to develop bioinformatics methods that allow efficient processing and analysis of large amounts of data generated in this technique. 


\section{REFERENCES}

1. Spener F, Lagarde M, Géloên A, Record M. What is lipidomics? Eur J Lipid Sci Technol. 2003;105(9):481-2. https:// doi.org/10.1002/ejlt.200390101.

2. Fahy E, Subramaniam S, Murphy RC, Nishijima M, Raetz CRH, Shimizu T, et al. Update of the LIPID MAPS comprehensive classification system for lipids. J Lipid Res. 2009;50(Suppl.): 9-14. https://doi.org/10.1194/jlr.R800095-JLR200.

3. Benjamins JA, Murphy EJ, Seyfried TN. Lipids. In: Brady ST, Siegel GJ, Albers RW, Price DL, editors. Basic Neurochemistry. Eighth Edi. New York: American Society for Neurochemistry; 2012. p. 81-100.

4. Antonelli L, Guarracino MR, Maddalena L, Sangiovanni M. Integrating imaging and omics data: A review. Biomed Signal Process Control. 2019;52:264-80. https://doi.org/10. 1016/j.bspc.2019.04.032.

5. Kishimoto K, Urade R, Ogawa T, Moriyama T. Nondestructive quantification of neutral lipids by thin-layer chromatography and laser-fluorescent scanning: Suitable methods for "lipidome" analysis. Biochem Biophys Res Commun. 2001; 281(3):657-62. https://doi.org/10.1006/bbrc.2001.4404.

6. Agatonovic-Kustrin S, Morton DW, Smirnov V, Petukhov A, Gegechkori V, Kuzina V, et al. Analytical Strategies in Lipidomics for Discovery of Functional Biomarkers from Human Saliva. Dis Markers. 2019. https://doi.org/10.1155/ 2019/6741518.

7. Hewelt-Belka W, Kot-Wasik A, Namieśnik J. Chemia analityczna w lipidomice. Anal Nauk i Prakt. 2014;1:4-12. Polish.

8. Han X, Yang K, Gross RW. Multi-dimensional mass spectrometry-based shotgun lipidomics and novel strategies for lipidomic analyses. Mass Spectrom Rev. 2012;31(1):134-78. https://doi.org/10.1002/mas.20342.

9. Isaac G, Jeannotte R, Esch SW, Welti R. New mass-spectrometry-based strategies for lipids. In: Setlow JK, editor. Genetic engineering. Boston, MA: Springer US; 2007. p. 129-57. https://doi.org/10.1007/978-0-387-34504-8_8.

10. World Health Organization [Internet]. 2018 [cited 2020 Oct 28]. The top 10 causes of death. Available from: https:// www.who.int/news-room/fact-sheets/detail/the-top-10causes-of-death.

11. Bikbov B, Purcell CA, Levey AS, Smith M, Abdoli A, Abebe M, et al. Global, regional, and national burden of chronic kidney disease, 1990-2017: a systematic analysis for the Global Burden of Disease Study 2017. Lancet. 2020;395:709-33. https://doi.org/10.1016/S0140-6736(20)30045-3.

12. National Institute of Diabetes and Digestive and Kidney Diseases [Internet]. 2016 [cited 2020 Oct 28]. Kidney Disease Statistics for the United States. Available from: https:// www.niddk.nih.gov/health-information/health-statistics/ kidney-disease.

13. Elshahat S, Cockwell P, Maxwell AP, Griffin M, O’Brien T, O'Neill C. The impact of chronic kidney disease on developed countries from a health economics perspective: A systematic scoping review. PLoS One. 2020;15(3):1-19. https:// doi.org/10.1371/journal.pone.0230512.

14. Afshinnia F, Rajendiran TM, Karnovsky A, Soni T, Wang X, Xie D, et al. Lipidomic Signature of Progression of Chronic Kidney Disease in the Chronic Renal Insufficiency Cohort. Kidney Int Reports. 2016;1(4):256-68. https://doi.org/10. 1016/j.ekir.2016.08.007.

15. Chen H, Chen L, Liu D, Chen DQ, Vaziri ND, Yu XY, et al. Combined Clinical Phenotype and Lipidomic Analysis Reveals the Impact of Chronic Kidney Disease on Lipid Metabolism. J Proteome Res. 2017;16(4):1566-78. https://doi. org/10.1021/acs.jproteome.6b00956.

16. Duranton F, Laget J, Gayrard N, Saulnier-Blache JS, Lundin U, Schanstra JP, et al. The CKD plasma lipidome varies with disease severity and outcome. J Clin Lipidol. 2019;13: 176-85. https://doi.org/10.1016/j.jacl.2018.07.010.

17. Afshinnia F, Rajendiran TM, Soni T, Byun J, Wernisch S, Sas KM, et al. Impaired B-oxidation and altered complex lipid fatty acid partitioning with advancing CKD. J Am Soc Nephrol. 2018;29(1):295-306. https://doi.org/10.1681/ ASN.2017030350.

18. Kordalewska M, Macioszek S, Wawrzyniak R, Sikorska-Wiśniewska M, Śledziński T, Chmielewski M, et al. Multiplat- 
form metabolomics provides insight into the molecular basis of chronic kidney disease. J Chromatogr B. 2019;1117:49-57. https://doi.org/10.1016/j.jchromb.2019.04.003.

19. Ferlay J, Colombet M, Soerjomataram I, Mathers C, Parkin DM, Piñeros M, et al. Estimating the global cancer incidence and mortality in 2018: GLOBOCAN sources and methods. Int J Cancer. 2019;144(8):1941-53. https://doi.org/ 10.1002/ijc.31937.

20. Pilleron S, Soto-Perez-de-Celis E, Vignat J, Ferlay J, Soerjomataram I, Bray F, et al. Estimated global cancer incidence in the oldest adults in 2018 and projections to 2050. Int J Cancer. 2020;148(3):601-8. https://doi.org/10.1002/ijc.33232.

21. Yu Z, Chen H, Ai J, Zhu Y, Li Y, Borgia JA, et al. Global lipidomics identified plasma lipids as novel biomarkers for early detection of lung cancer. Oncotarget. 2017;8(64):107899906. https://doi.org/10.18632/oncotarget.22391.

22. Ros-Mazurczyk M, Jelonek K, Marczyk M, Binczyk F, Pietrowska M, Polanska J, et al. Serum lipid profile discriminates patients with early lung cancer from healthy controls. Lung Cancer. 2017;112:69-74. https://doi.org/10.1016/j.lung can.2017.07.036.

23. Noreldeen HAA, Du L, Li W, Liu X, Wang Y, Xu G. Serum lipidomic biomarkers for non-small cell lung cancer in nonsmoking female patients. J Pharm Biomed Anal. 2020; 185:113220. https://doi.org/10.1016/j.jpba.2020.113220.

24. Lee G Bin, Lee JC, Moon MH. Plasma lipid profile comparison of five different cancers by nanoflow ultrahigh performance liquid chromatography-tandem mass spectrometry. Anal Chim Acta. 2019;1063:117-26. https:/doi. org/10.1016/j.aca.2019.02.021.

25. Cho YT, Su H, Chiang YY, Shiea J, Yuan SSF, Hung WC, et al. Fine Needle Aspiration Combined With Matrix-assisted Laser Desorption Ionization Time-of-Flight/Mass Spectrometry to Characterize Lipid Biomarkers for Diagnosing Accuracy of Breast Cancer. Clin Breast Cancer. 2017;17(5):37381. https://doi.org/10.1016/j.clbc.2017.04.014.

26. Chen X, Chen H, Dai M, Ai J, Li Y, Mahon B, et al. Plasma lipidomics profiling identified lipid biomarkers in distinguishing early-stage breast cancer from benign lesions. Oncotarget. 2016;7(24):36622-31. https://doi.org/10. 18632/oncotarget.9124.

27. Yin M, Tan S, Li X, Hou Y, Cao G, Li K, et al. Identification of phosphatidylcholine and lysophosphatidylcholine as novel biomarkers for cervical cancers in a prospective cohort study. Tumor Biol. 2016;37:5485-92. https://doi.org/ 10.1007/s13277-015-4164-X.

28. Skotland T, Ekroos K, Kauhanen D, Simolin H, Seierstad T, Berge V, et al. Molecular lipid species in urinary exosomes as potential prostate cancer biomarkers. Eur J Cancer. 2017; 70:122-32. https://doi.org/10.1016/j.ejca.2016.10.011

29. Buszewska-Forajta M, Raczak-Gutknecht J, Artymowicz M, Wesołowski W, Buczkowski K, Iżycka-Świeszewska E, et al. The potential role of fatty acids in prostate cancer determined by GC-MS analysis of formalin-fixed paraffin-embedded tissue samples. J Pharm Biomed Anal. 2021;196. https://doi.org/10.1016/j.jpba.2021.113907.

30. Encyclopædia Britannica. [Internet]. 2019 [cited 2020 Oct 30]. Enns G. Metabolic disease. Available from: https:// www.britannica.com/science/metabolic-disease 3907

31. National Center for Biotechnology Information. [Internet]. [cited 2020 Oct 30]. Nutritional and Metabolic Diseases.Available from: https://www.ncbi.nlm.nih.gov/books/NBK22259/.

32. Araszkiewicz A, Bandurska-Stankiewicz E, Budzyński A, Cypryk K, Czech A, Czupryniak L, et al. 2019 Guidelines on the management of diabetic patients. A position of Diabetes Poland. Clin Diabetol. 2019;8(1):1-95. https:/doi.org/ 10.5603/dk.2019.0001.

33. Drągowski P, Czyżewska U, Cekała E, Lange P, Zadykowicz R, Sójka A, et al. Diabetes As a Social and Economic Problem. Pol Przegląd Nauk o Zdrowiu. 2014;2(39):163-6. Polish.

34. World Health Organization. [Internet]. 2020 [cited 2020 Oct 28]. Diabetes. Available from: https:/www.who.int/ news-room/fact-sheets/detail/diabetes.

35. International Diabetes Federation. IDF DIABETES ATLAS 9th edition 2019 Global Fact Sheet. International Diabetes Federation Diabetes Atlas, Ninth Edition 2019. 
36. Association American Diabetes. 2. Classification and Diagnosis of Diabetes. Diabetes Care. 2017 Jan 1;40(Suppl. 1): 11-24. https://doi.org/10.2337/dc17-S005.

37. Dall TM, Yang W, Gillespie K, Mocarski M, Byrne E, Cintina I, et al. The economic burden of elevated blood glucose levels in 2017: Diagnosed and undiagnosed diabetes, gestational diabetes mellitus, and prediabetes. Diabetes Care. 2019;42(9):1661-8. https://doi.org/10.2337/dc18-1226.

38. Tulipani S, Palau-Rodriguez M, Miñarro Alonso A, Cardona F, Marco-Ramell A, Zonja B, et al. Biomarkers of Morbid Obesity and Prediabetes by Metabolomic Profiling of Human Discordant Phenotypes. Clin Chim Acta. 2016;463:5361. https://doi.org/10.1016/j.cca.2016.10.005.

39. Zeng Y, Mtintsilana A, Goedecke JH, Micklesfield LK, Olsson T, Chorell E. Alterations in the metabolism of phospholipids, bile acids and branched-chain amino acids predicts development of type 2 diabetes in black South African women: a prospective cohort study. Metabolism. 2019;95:57-64. https://doi.org/10.1016/j.metabol.2019.04.001

40. Wang-Sattler R, Yu Z, Herder C, Messias AC, Floegel A, He Y, et al. Novel biomarkers for pre-diabetes identified by metabolomics. Mol Syst Biol. 2012;8. https://doi.org/10.1038/ msb.2012.43.

41. Zeng H, Tong R, Tong W, Yang Q, Qiu M, Xiong A, et al. Metabolic Biomarkers for Prognostic Prediction of Pre-diabetes: results from a longitudinal cohort study. Sci Rep. 2017;7(1):112. https://doi.org/10.1038/s41598-017-06309-6.

42. Yan L, Han P, Man J, Tian Y, Wang F, Wang J. Discovery of lipid profiles of type 2 diabetes associated with hyperlipidemia using untargeted UPLC Q-TOF/MS-based lipidomics approach. Clin Chim Acta. 2021;520(May):53-62. https:// doi.org/10.1016/j.cca.2021.05.031.

This work is available in Open Access model and licensed under a Creative Commons Attribution-NonCommercial 3.0 Poland License - http://creativecommons.org/ licenses/by-nc/3.0/pl/deed.en. 